Case Report

\title{
Toxic epidermal necrolysis induced by acetaminophen: a case report
}

\author{
M. C. Gupta, Niti Mittal*, Nishikant Sharma
}

Department of Pharmacology, Pt. B D

Sharma Postgraduate Institute of

Medical Sciences, Rohtak 124001,

Haryana, India

Received: 23 August 2013

Accepted: 3 September 2013

*Correspondence to:

Dr. Niti Mittal,

Email:drniti.mittal@gmail.com

C 2013 Gupta MC et al. This is an open-access article distributed under the terms of the Creative Commons Attribution Non-Commercial License, which permits unrestricted noncommercial use, distribution, and reproduction in any medium, provided the original work is properly cited.

\begin{abstract}
Acetaminophen is a very commonly used analgesic and antipyretic drug across various age groups. Although mild to moderate cutaneous reactions have been reported quite frequently, serious reactions like Stevens -Johnson syndrome and Toxic epidermal necrolysis (TEN) are very rare. We report the case of a 10 year old child who had TEN after ingestion of tablet acetaminophen. This case report highlights the need to be critically aware of this rare and serious adverse effect of this commonly used over the counter drug.
\end{abstract}

Keywords: Acetaminophen, Toxic epidermal necrolysis, Causality

\section{INTRODUCTION}

Acetaminophen (paracetamol) is among one of the very commonly used drugs in different populations due to its analgesic and antipyretic actions. It is considered to be very safe and well tolerated drug when given in isolation and in appropriate therapeutic dosages. The incidence of cutaneous adverse reactions reported with standard dosages is quite rare. ${ }^{1}$ Several cases of urticaria, fixed drug eruptions, maculopapular eruptions etc. have been reported. $^{2}$ However, serious cutaneous reactions like erythema multiforme, Stevens -Johnson syndrome and Toxic epidermal necrolysis (TEN) are extremely rare considering the enormous consumption of this drug. The reported incidence in pediatric population is even lower. We report a case of TEN in a 10 year old female child after acute ingestion of acetaminophen.

\section{CASE REPORT}

A 10 year old female child (body weight: $21 \mathrm{~kg}$ ) presented to the outpatient department with chief complaint of moderate to high grade fever for 1 day. No localising symptoms/signs were observed and she was prescribed tablet acetaminophen $500 \mathrm{mg}$ twice daily orally by the treating physician. Within 10 minutes of taking first dose, child developed itching all over the body. On repeating the second and third doses in evening and next morning she complained of small fluid filled lesions all over the body. The fever also continued. The child presented to the hospital and on cutaneous examination, generalised vesicular and bullous eruption associated with extensive epidermal detachment (49 percent body surface area involved), skin necrosis and positive Nikolsky's sign were recorded. Oral and genital mucosa had ulcerative lesions and ocular examination showed serous discharge and crusting of eyelids. The past medical history revealed that she had two similar episodes one and a half year back also after the intake of a syrup containing ibuprofen and acetaminophen (Syrup Combiflam) for fever. The child got treatment at a private hospital but no records were available.

Based on history and typical clinical features, she was diagnosed with toxic epidermal necrolysis (TEN) and hospitalised. Routine laboratory investigations including haemogram, hepatic and renal function tests, and urine microscopic examination were performed and were within normal limits. The drug was withheld and she was given supportive care (intravenous fluids and non adhesive wound dressings) and systemic steroid therapy (intravenous hydrocortisone $50 \mathrm{mg}$ twice daily). The 
lesions subsided, child showed gradual improvement and after 10 days of hospitalisation, she was discharged in a satisfactory condition, without any sequelae.

\section{DISCUSSION}

This is a case of TEN occurring in a 10 year old female child after acute ingestion of acetaminophen. The child was not on any other medication and no alternative etiology was present. History of two similar episodes in the past after ingestion of acetaminophen/ibuprofen combination, although undocumented, further strengthens the causal association of acetaminophen with the event. Causality assessment was carried out using WHO-UMC and Naranjo's causality assessment scales (score 7) both of which showed a probable causal association of acetaminophen with the adverse event. Rechallenge was not done due to ethical concerns.

Acetaminophen is among rarely implicated drugs in the causation of TEN, especially among pediatric population. Till now, there have been two such reports in children. ${ }^{3,4}$ Another case of TEN with combination of acetaminophen and metoclopramide in a 42 year old woman has been reported. ${ }^{5}$ A case control study also demonstrated increased risk of Stevens-Johnson syndrome and TEN with acetaminophen. $^{6}$

\section{CONCLUSION}

Acetaminophen is a very rampantly used drug in adult as well as pediatric patients due to its relatively safe clinical profile. TEN is among rare adverse events reported with this drug. However, physicians need to be aware of this rare side effect in order to provide appropriate management to the sufferer. Also, any such case observed should be reported in order to generate further data pertaining to risk factors involved and incidence in general.

\section{Funding: None \\ Conflict of interest: None declared \\ Ethical approval: Not required}

\section{REFERENCES}

1. Kvedariene V, Bencherioua AM, Messaad D, Godard $\mathrm{P}$, Bousquet J, Demoly P. The accuracy of the diagnosis of suspected paracetamol (acetaminophen) hypersensitivity: results of a single-blinded trial. Clin Exp Allergy 2002;32:1366-9.

2. Ibañez MD, Alonso E, Muñoz MC, Martinez E, Laso MT. Delayed hypersensitivity reaction to paracetamol (acetaminophen). Allergy 1996;51:121-3.

3. Bygum A, Gregersen JW, Buus SK. Acetaminopheninduced toxic epidermal necrolysis in a child. Pediatr Dermatol. 2004;21:236-8.

4. Halevi A, Ben-Amitai D, Garty BZ. Toxic epidermal necrolysis associated with acetaminophen ingestion. Ann Pharmacother 2000;34:32-4.

5. Rajagopalan S, Kaur S, Dogra S, Shafiq N, Bhalla A, Pandhi P, et al. Toxic Epidermal Necrolysis induced by rarely implicated drugs. Indian $\mathrm{J}$ Pharmacol 2012;44:272-3.

6. Roujeau JC, Kelly JP, Naldi L, Rzany B, Stern RS, Anderson T, et al. Medication use and the risk of Stevens-Johnson syndrome or toxic epidermal necrolysis. N Engl J Med 1995;333:1600-7.

doi:10.5455/2319-2003.ijbcp20131232

Cite this article as: Gupta MC, Mittal N, Sharma N.

Toxic epidermal necrolysis induced by acetaminophen: a case report. Int J Basic Clin Pharmacol 2013;2:831-2. 